\title{
On the "direct" calculation of thermal rate constants
}

\author{
Ward H. Thompson and William H. Miller \\ Department of Chemistry, University of California, and Chemical Sciences Division, Lawrence Berkeley \\ Laboratory, Berkeley, California 94720
}

(Received 23 December 1994; accepted 7 February 1995)

\begin{abstract}
We present a new approach for the direct (and correct) calculation of thermal rate constants $k(T)$ ("direct" meaning that one avoids having to solve the state-to-state reactive scattering problem, and "correct" meaning that the method contains no inherent approximations). The rate constant is obtained from the long time limit of the flux-position correlation function, $C_{f, s}(t)$, whose calculation is made efficient by taking advantage of the low rank of the flux operator. Specifically, the trace required to obtain $C_{f, s}(t)$ is evaluated by a Lanczos iteration procedure which calculates only the nonzero eigenvalues. The propagation in complex time, $t_{c}=t-i \hbar \beta / 2$, is carried out using a Chebychev expansion. This method is seen to be both accurate and efficient by application to the Eckart barrier, the collinear $\mathrm{H}+\mathrm{H}_{2}$ reaction, and the three-dimensional $\mathrm{D}+\mathrm{H}_{2} \quad(J=0)$ reaction. (C) 1995 American Institute of Physics.
\end{abstract}

\section{INTRODUCTION}

A long-standing goal of our research group has been the development of ways to calculate rate constants for chemical reactions directly, i.e., without having to solve the complete state-to-state reactive scattering problem, yet still correctly, i.e., without inherent approximation. That is, we seek to develop rigorous theoretical approaches that are the outgrowth of ideas from transition state theory (TST), which is a very useful (though approximate) approach to determining rate constants "directly."

Our initial efforts ${ }^{1,2}$ in this direction focused on the thermally averaged rate constant $k(T)$ for bimolecular reactions; a formally exact quantum expression for the rate was derived, and this was useful, for example, in showing the nature of the TST approximation and in suggesting more accurate quantum mechanical versions of TST. Later, ${ }^{3}$ this rigorous rate expression was written in terms of reactive flux autocorrelation functions (that are similar, though not identical to earlier such expressions obtained by Yamamoto ${ }^{4}$ ), and a number of groups ${ }^{3,5-19}$ (including ours) have used this formulation as the basis for practical calculations.

More recently our attention shifted to the "direct" calculation of the cumulative reaction probability (CRP), $N(E)$, which is defined as

$$
N(E)=\sum_{\mathbf{n}_{p}, \mathbf{n}_{r}}\left|S_{\mathbf{n}_{p}, \mathbf{n}_{r}}(E)\right|^{2},
$$

and in terms of which the thermal rate constant is given by

$$
k(T)=\frac{1}{2 \pi \hbar Q_{r}(T)} \int_{0}^{\infty} d E e^{-\beta E} N(E),
$$

where $Q_{r}$ is the reactant partition function per unit volume and $\beta=1 / k_{b} T$. \{Sometimes, usually for unimolecular reactions, one is interested in the microcanonical rate, $k(E)$, the average rate for a given total energy, and it is given in terms of the CRP by

$$
k(E)=\left[2 \pi \hbar \rho_{r}(E)\right]^{-1} N(E),
$$

where $\rho_{r}$ is the density of reactant states per unit energy.\} Equation (1.1) is of course not a "direct" expression for the CRP since it requires the $S$-matrix elements for transitions from all the energetically open reactant states $\left\{\mathbf{n}_{r}\right\}$ to all such product states $\left\{\mathbf{n}_{p}\right\}$. The following "direct" expression for the CRP was obtained, ${ }^{3}$ however, as a by-product of the flux correlation function analysis,

$$
N(E)=\frac{1}{2}(2 \pi \hbar)^{2} \operatorname{tr}[\hat{F} \delta(E-\hat{H}) \hat{F} \delta(E-\hat{H})],
$$

where $\hat{F}$ is a flux operator (defined with respect to a dividing surface which separates reactants from products) and $\hat{H}$ is the total Hamiltonian of the system. A practical implementation of Eq. (1.4) was achieved ${ }^{20,21}$ by representing the microcanonical density operator as

$$
\delta(E-\hat{H})=-\frac{1}{\pi} \operatorname{Im}(E+i \hat{\boldsymbol{\epsilon}}-\hat{H})^{-1},
$$

where $\hat{\epsilon}$ is a potential energy operator which enforces outgoing wave boundary conditions. Such absorbing (empirical optical, negative imaginary) potentials have been used by a number of workers ${ }^{22-27}$ in a variety of contexts. A very efficient computational procedure ${ }^{28}$ based on this approach has been developed and applied to several challenging problems, e.g., a full dimensional calculation of $N(E)$ for the $\mathrm{H}_{2}+\mathrm{OH} \rightarrow \mathrm{H}_{2} \mathrm{O}+\mathrm{H}$ reaction. ${ }^{29}$ \{We note that another useful implementation of Eq. (1.4) has been achieved ${ }^{30}$ by representing the microcanonical density operator as

$$
\delta(E-\hat{H})=\left(\frac{\alpha M}{\pi}\right)^{1 / 2}\left[e^{-\alpha(\hat{H}-E)^{2}}\right]^{M},
$$

for $\alpha$ sufficiently small and $M$ sufficiently large.\}

In the present paper we return to the problem of the direct determination of the thermal rate constant $k(T)$, for three reasons. First, if one wishes to have $k(T)$ itself, and is not primarily interested in $N(E)$, as is typically the case for bimolecular reactions, then it is clearly desirable to be able to compute it for the temperature of interest and not have to compute $N(E)$ over a range of $E$ in order to carry out the Boltzmann average in Eq. (1.2). 
A second reason for refocusing on $k(T)$ is that its calculation (vide infra) is able to avoid introducing the absorbing potential $\hat{\epsilon}$ discussed above regarding Eq. (1.5). Although the use of absorbing potentials has made it possible to carry out the $N(E)$ calculations noted above, their use does introduce numerical convergence parameters into the calculation that one would be quite happy to avoid. The $k(T)$ calculation can bypass the use of absorbing potentials because it is carried out in the time domain and thus involves the time evolution operator, $\exp (-i \hat{H} t / \hbar)$, whereas the energy domain calculation of $N(E)$ involves the Green's function $G^{+}(E)$. These two operators are related by

$$
\begin{aligned}
G^{+}(E) & =(E+i \hat{\epsilon}-\hat{H})^{-1} \\
& =(i \hbar)^{-1} \int_{0}^{\infty} d t e^{i(E+i \hat{\epsilon}-\hat{H}) t / \hbar},
\end{aligned}
$$

and one sees that the absorbing potential $\hat{\epsilon}$ is needed so that the $t \rightarrow \infty$ part of the integrand in Eq. (1.7b) is damped; i.e., it provides a long time cutoff. When carrying out the $t$-space calculation for $k(T)$, however, it is easy to incorporate a long time cutoff without using absorbing potentials-one simply stops the calculation at finite $t$.

The third reason that we have returned to the calculation of $k(T)$ is that some of the tricks that have been learned from doing the $N(E)$ calculation "directly" can be carried over to the $k(T)$ calculation and provide a more powerful approach than earlier ones. In particular, we evaluate the quantum mechanical trace expression for $k(T)$ (vide infra) using an iterative Lanczos procedure analogous to that used by Manthe et $a l .{ }^{28}$ for the $N(E)$ calculation. The low rank of the relevant operator greatly reduces the number of operations of the time evolution operator that are required.

Section II first summarizes the relevant flux correlation expressions for $k(T)$ that we use. Section III describes the computational details of the present method, and then Sec. IV presents results for the one-dimensional Eckart barrier, the collinear $\mathrm{H}+\mathrm{H}_{2}$ reaction, and the three-dimensional $\mathrm{D}+\mathrm{H}_{2}$ reaction for $J=0$. We compare to previous exact calculations and discuss the accuracy of the present method. Section V concludes.

\section{FLUX CORRELATION FUNCTIONS: REVIEW OF THE RELEVANT FORMULAS}

We begin with the expression for the exact thermal rate constant derived previously by one of us ${ }^{1}$ in terms of a quantum mechanical trace,

$$
k(T)=\frac{1}{Q_{r}} \operatorname{tr}\left(e^{-\beta \hat{H}} \hat{F} \hat{\wp}\right) .
$$

Here $Q_{r}$ is the reactant partition function, $\hat{F}$ is the symmetrized flux operator [in the original formulation $k(T)$ is given as the real part of the right-hand side of Eq. (2.1) with the unsymmetrized flux operator], and $\hat{\wp}$ is a projection operator onto "reactive space." This expression is very intuitive in that it gives the rate constant as a thermal average of the reactive flux through a surface dividing reactants and products. This is analogous to the expression for the exact classical rate constant,

$$
\begin{aligned}
k_{\mathrm{CL}}(T)= & \frac{1}{Q_{r} h^{F}} \int d \mathbf{p} \int d \mathbf{q} e^{-\beta H(\mathbf{p}, \mathbf{q})} \delta[f(\mathbf{q})] \\
& \times \frac{\partial f(\mathbf{q})}{\partial \mathbf{q}} \cdot \frac{\mathbf{p}}{m} \chi(\mathbf{p}, \mathbf{q})
\end{aligned}
$$

which is the thermal average of the classical reactive flux through a surface $f(\mathbf{q})=0$ dividing reactants from products. $\chi(\mathbf{p}, \mathbf{q})$ is the "characteristic function" which takes the value 1 for reactive trajectories and 0 for nonreactive trajectories. This plays the same role in the classical expression as does $\hat{\wp}$ in the quantum mechanical expression, Eq. (2.1), which projects onto the reactive part of the Hilbert space.

As noted by Tromp and Miller, ${ }^{5}$ the projection operator can be represented in many ways. For the purpose of this discussion, we consider a one-dimensional barrier problem with the barrier at $s=0$. The reactants are defined by $s<0$ and products by $s>0$, and $\hat{p}$ is the momentum conjugate to $s$. In the original formulation the projection operator was taken to be

$$
\hat{\wp}=\lim _{t \rightarrow \infty} e^{-i \hat{H} t / \hbar} h(\hat{p}) e^{i \hat{H} t / \hbar},
$$

where $h$ is the Heaviside step function, $h(\xi)=1$ for $\xi>0$ and $h(\xi)=0$ otherwise. This operator selects out those components of the basis which have momentum in the positive direction at $t \rightarrow-\infty$. Miller, Schwartz, and Tromp ${ }^{3}$ showed that this projection operator and one based on the position step function,

$$
\hat{\wp}=\lim _{t \rightarrow \infty} e^{-i \hat{H} t / \hbar} h(-\hat{s}) e^{i \hat{H} t / \hbar},
$$

are equivalent in the long time limit indicated. This latter projection operator selects out those components of the basis which were on the reactant side of the barrier at $t \rightarrow-\infty$. Clearly both of these operators project onto the reactive space.

Using the projection operators in Eqs. (2.3) and (2.4), and the property $[\hat{H}, \hat{\wp}]=0$, the exact thermal rate constant can be expressed as

$$
k(T)=\frac{1}{Q_{r}} \lim _{t \rightarrow \infty} \operatorname{tr}\left[h(\hat{p}) e^{i \hat{H}_{c}^{*} / \hbar} \hat{F} e^{-i \hat{H} t_{c} / \hbar} h(\hat{p})\right],
$$

or equivalently as

$$
k(T)=\frac{1}{Q_{r}} \lim _{t \rightarrow \infty} \operatorname{tr}\left[h(-\hat{s}) e^{i \hat{H} t_{c}^{*} / \hbar} \hat{F} e^{-i \hat{H} t_{c} / \hbar} h(-\hat{s})\right],
$$

where we have combined the propagators with the Boltzmann operator to obtain a single propagator in complex time, $t_{c}=t-i \beta \hbar / 2$, and also used the property of the step function $h(\xi) * h(\xi)=h(\xi)$ in order to express $k(T)$ as the trace of an Hermitian operator. From these expressions we define the flux-momentum correlation function

$$
C_{f p}(t)=\operatorname{tr}\left[h(\hat{p}) e^{i \hat{H} t_{c}^{*} / \hbar} \hat{F} e^{-i \hat{H}_{c} / \hbar} h(\hat{p})\right],
$$

and the flux-position correlation function

$$
C_{f s}(t)=\operatorname{tr}\left[h(-\hat{s}) e^{i \hat{H} t_{c}^{*} / \hbar} \hat{F} e^{-i \hat{H} t_{c} / \hbar} h(-\hat{s})\right],
$$

so that the thermal rate constant is given by 


$$
k(T)=\frac{1}{Q_{r}} \lim _{t \rightarrow \infty} C_{f p}(t)=\frac{1}{Q_{r}} \lim _{t \rightarrow \infty} C_{f s}(t) .
$$

In addition to these correlation functions Miller, Schwartz, and Tromp ${ }^{3}$ derived a flux-flux autocorrelation function

$$
C_{f f}(t)=\operatorname{tr}\left(\hat{F} e^{i \hat{H} t_{c}^{*} / \hbar} \hat{F} e^{-i \hat{H} t_{c} / \hbar}\right),
$$

and a left-right correlation function

$$
C_{s s}(t)=\operatorname{tr}\left[h(-\hat{s}) e^{i \hat{H}_{c}^{*} / \hbar} h(\hat{s}) e^{-i \hat{H} t_{c} / \hbar} h(-\hat{s})\right],
$$

in terms of which the rate constant can also be expressed,

$$
k(T)=\frac{1}{Q_{r}} \int_{0}^{\infty} C_{f f}(t) d t=\frac{1}{Q_{r}} \lim _{t \rightarrow \infty} \frac{d}{d t} C_{s s}(t) .
$$

One expects the flux-position and flux-momentum correlation functions to be most efficient since one only needs to evaluate them at a single (long) time (see Sec. III B). The flux-flux autocorrelation function must be evaluated at many times in order to compute its integral, and the left-right correlation function needs to be evaluated at two or more times in order to obtain the derivative.

\section{DETAILS OF CALCULATION}

\section{A. Evaluating the trace}

The flux operator is of low rank; in one dimension, diagonalizing it in a finite basis representation yields only two nonzero eigenvalues, one negative and one positive, corresponding to flux in the forward and backward directions. ${ }^{13,14,31}$ The low rank of $\hat{F}$ implies a similar low rank for the operator $\hat{C}_{f s}(t)$,

$$
\hat{C}_{f s}(t)=h(-\hat{s}) e^{i \hat{H} t_{c}^{*} / \hbar} \hat{F} e^{-i \hat{H} t_{c} / \hbar} h(-\hat{s}),
$$

the trace of which is the rate constant (for large enough $t$ ). In the general multidimensional case we expect the number of nonzero eigenvalues of $\hat{C}_{f s}(t)$ (i.e., its rank) to be approximately the number of states of the activated complex of TST that would contribute significantly to the partition function of the activated complex.

The situation is thus analogous to Manthe and Miller's ${ }^{28}$ treatment of the microcanonical case, where the CRP $N(E)$ was expressed as the trace of a matrix/operator of low rank, the number of nonzero eigenvalues being approximately the number of energetically accessible states of the activated complex of TST. We thus follow the same strategy as Manthe and Miller ${ }^{28}$ and use a Lanczos iterative procedure to compute the trace of $\hat{C}_{f s}(t)$, for the number of Lanczos iterations required for convergence will only be a few more than the rank of the matrix.

In the Lanczos procedure one starts with some random vector $\mathbf{v}$ and builds a Krylov basis by multiplying $\hat{C}_{f s}(t)$ successively onto $\mathbf{v}$, i.e.,

$$
\begin{aligned}
& \mathbf{v}_{0}=\mathbf{v}, \\
& \mathbf{v}_{1}=\mathbf{C}_{f s}(t) \cdot \mathbf{v}_{0}+\mathrm{SO} \\
& \mathbf{v}_{2}=\mathbf{C}_{f s}(t) \cdot \mathbf{v}_{1}+\mathrm{SO},
\end{aligned}
$$

etc., where "SO" implies Schmidt orthogonalization to all preceeding vectors. The matrix of $\hat{C}_{f s}(t)$ in this basis (which is produced "automatically" in the process of constructing the basis) is tridiagonal. When the size of the Krylov basis (i.e., the number of Lanczos iterations) exceeds the rank of the matrix, then no new basis functions are introduced by further iterations, and the eigenvalues (and therefore the trace) of the matrix in the Krylov basis are the exact ones (i.e., the same as those in the original, perhaps much larger basis).

\section{B. Propagation in complex time}

When applying the operator/matrix $\hat{C}_{f s}(t)$ to a vector, each operator in $\hat{C}_{f s}(t)$ operates sequentially (from the right). Thus two operations of the time evolution operator-by far the most time consuming part of the calculation- $\exp \left(-i \hat{H} t_{c} / \hbar\right)$ and $\exp \left(i \hat{H} t_{c}^{*} / \hbar\right)$, are required for each operation of $\hat{C}_{f s}(t)$.

In this paper we have used the Chebychev polynomial expansion of the propagator ${ }^{32}$

$$
\begin{aligned}
e^{-i \mathbf{H} t_{c} / \hbar} \cong & e^{-i \bar{H} t_{c} / \hbar} \sum_{n=0}^{N_{c}}\left(2-\delta_{n, 0}\right) i^{-n} J_{n}\left(\frac{\Delta H t_{c}}{2 \hbar}\right) \\
& \times T_{n}\left(\frac{\mathbf{H}-\bar{H}}{\Delta H / 2}\right),
\end{aligned}
$$

where $\mathbf{H}$ is the Hamiltonian matrix in some finite basis, $N_{c}$ is the order of the highest Chebychev polynomial, the $J_{n}$ are Bessel functions, and the $T_{n}$ are the Chebychev polynomials obtained by the recursion relation

$$
T_{n+1}(x)=2 x T_{n}(x)-T_{n-1}(x) .
$$

$\Delta H$ is the spectral range of the Hamiltonian and $\bar{H}$ is the average value of the Hamiltonian. Specifically, if $\lambda_{\max }$ and $\lambda_{\text {min }}$ are the largest and smallest eigenvalues of $\mathbf{H}$, then

$$
\Delta H=\lambda_{\max }-\lambda_{\min },
$$

and

$$
\bar{H}=\frac{\lambda_{\max }+\lambda_{\min }}{2} .
$$

In cases where the Hamiltonian can be stored in core memory, we explicitly diagonalize the Hamiltonian to obtain $\lambda_{\min }$ and $\lambda_{\max }$. When this is not the case (as for the $\mathrm{D}+\mathrm{H}_{2}$ reaction in Sec. IV C), we estimate $\lambda_{\min }$ and $\lambda_{\max }$ from a low order Lanczos calculation using a sparse Hamiltonian matrix multiply.

The number of Chebychev polynomials needed depends on the spectral range and the propagation time. This relation occurs because the Bessel functions become exponentially damped as the order $n$ becomes larger than the argument. In our case this implies the guideline

$$
N_{c}>\frac{\Delta H\left|t_{c}\right|}{2 \hbar} .
$$

The Chebychev expansion for the propagator provides several advantages. It is an efficient representation and allows one to combine the operation of the time evolution 
operator and the Boltzmann operator. Intermediate results and restarting are not required. We are primarily interested in the case of large multidimensional systems where the Hamiltonian matrix cannot be stored directly. The Chebychev propagation only requires the storage of three complex vectors, and one can make use of a sparse matrix multiplication routine for applying the Hamiltonian matrix onto a vector.

\section{The flux operator}

The flux through a dividing surface defined by $f(\mathbf{q})=0$ is given by

$$
\hat{F}=\frac{1}{2}\left\{\delta[f(\mathbf{q})] \mathbf{n}_{f} \cdot \hat{\mathbf{p}}+\hat{\mathbf{p}} \cdot \mathbf{n}_{f} \delta[f(\mathbf{q})]\right\},
$$

where $\mathbf{n}_{f}$ is the unit vector normal to the dividing surface and $\hat{\mathbf{p}}$ is the momentum operator. However, this flux operator may be equivalently expressed as

$$
\hat{F}=\frac{i}{\hbar}[\hat{H}, h(f(\mathbf{q}))] .
$$

We note that these two expressions for the flux operator do not have identical numerical properties in an $L^{2}$ basis representation. We have chosen to use the form in Eq. (3.8) because it is more straightforwardly generalized to higher dimensions and is easily applied with a sparse matrix multiply routine as mentioned above.

\section{The basis set}

We have chosen to use a discrete variable representation ${ }^{33-35}$ as our finite basis. Specifically in the examples shown in Sec. IV we have used the sinc function DVR of Colbert and Miller ${ }^{35}$ in all cases except for the Jacobi angle in the three-dimensional $\mathrm{D}+\mathrm{H}_{2}$ reaction. For the Jacobi angle we have used a symmetrized Gauss-Legendre DVR. The sinc function DVR has evenly spaced points with the grid spacing $\Delta x$ determined by the maximum kinetic energy in the problem. We have thus used the thermal de Broglie wavelength and a grid constant, $N_{B}$, to determine $\Delta x$,

$$
\Delta x=\frac{2 \pi}{N_{B}}\left(\frac{2 \mu k_{b} T}{\hbar^{2}}\right)^{-1 / 2} .
$$

For the present applications we have found $N_{B}=10-14$ to be sufficiently large. The $1 \mathrm{D}$ kinetic energy matrix elements in a sinc function DVR can be expressed in closed form. ${ }^{35}$ In addition, in the DVR the potential energy is approximated as a diagonal matrix with the diagonal elements equal to the potential evaluated at the specified DVR point. The greatest advantage, however, is the fact that the Hamiltonian matrix for a multidimensional system is sparse. This allows one to use a sparse matrix multiplication of the Hamiltonian within the Chebychev algorithm when the size of the matrix is too large to be stored in the core memory of the computer.

In the present formulation we need to evaluate the DVR matrix elements of the flux operator, $\hat{F}$. The flux operator is easily evaluated in the form described in Sec. III C. That is, the matrix elements of the flux operator in the DVR are given by

$$
\mathbf{F}_{j, j^{\prime}}=\frac{i}{\hbar} \mathbf{T}_{j, j^{\prime}}\left[h\left(s_{j^{\prime}}\right)-h\left(s_{j}\right)\right],
$$

where $h\left(s_{j}\right)$ is the step function evaluated at the $j$ th DVR point, and $\mathbf{T}_{j, j^{\prime}}$ is the kinetic energy matrix.

\section{RESULTS}

In order to demonstrate the utility of the present method we have applied it to three test cases, the one-dimensional Eckart barrier, the two-dimensional collinear $\mathrm{H}+\mathrm{H}_{2}$ reaction, and the three-dimensional $\mathrm{D}+\mathrm{H}_{2}$ reaction for $J=0$.

\section{A. The Eckart barrier}

As a starting point, we have applied the present method to the one-dimensional Eckart barrier,

$$
V(s)=V_{0} \operatorname{sech}^{2}(s / a),
$$

with $V_{0}=0.425 \mathrm{eV}, a=0.734$ a.u., and a mass of 1061 a.u. These parameters model the collinear $\mathrm{H}+\mathrm{H}_{2}$ reaction. The basis is specified by $N_{B}$ and $Q_{\max } \cdot Q_{\max }$ defines the extent of the basis; the DVR grid is truncated for $|s|>Q_{\max }$.

Below we investigate the efficiency of the flux-position correlation functions to compute thermal rate constants. For completeness, we have tested the Lanczos scheme for computing rate constants using the flux-flux, left-right, and flux-momentum correlation functions. The number of nonzero eigenvalues is roughly the same in all cases. Therefore, using $C_{f f}(t)$ and $C_{s s}(t)$ is less efficient since they must be computed at more than one time. We have found the fluxmomentum correlation function to have poorer convergence properties than $C_{f s}(t)$, making it less desirable.

For simple barrier crossing reactions, such as the present examples, one expects that $C_{f s}(t)$ will reach a constant value (a "plateau") at times on the order of $\hbar \beta$, giving the correct rate constant. At longer times, reflection from the edge of the grid gives spurious results. Thus, we determine the real propagation time, $t$, by specifying a unitless time factor, $\tau$, according to the relation

$$
t=\tau \hbar \beta .
$$

The number of Chebychev terms, $N_{c}$, depends on the propagation time and can also be specified by a single factor, $\eta$,

$$
N_{c}=\eta \frac{\Delta H\left|t_{c}\right|}{2 \hbar} .
$$

In order to evaluate the efficiency of the method, we are interested in examining (1) the time needed to reach the plateau value of the correlation function, (2) the grid size necessary to obtain a reasonably wide plateau period, (3) the number of Chebychev terms needed in the expansion, and particularly important, (4) the number of Lanczos iterations necessary to converge the rate constant.

Figure 1 shows the rate constant obtained from $C_{f s}(t)$ as a function of $t$ for (a) $T=200 \mathrm{~K}$, (b) $T=300 \mathrm{~K}$, and (c) $T=1000 \mathrm{~K}$ for different grid sizes $\left[k(T ; t)=C_{f s}(t) / Q_{r}(T)\right]$. Note that the plateau begins around $25 \mathrm{fs}$ for $T=200 \mathrm{~K}$ and around $18 \mathrm{fs}$ for $T=300 \mathrm{~K}$, while for these temperatures $\hbar \beta \sim 38 \mathrm{fs}$ and $25 \mathrm{fs}$, respectively. In contrast, for $T=1000 \mathrm{~K}$ the plateau occurs at about $t=10$ fs while $\hbar \beta \sim 7$. This be- 

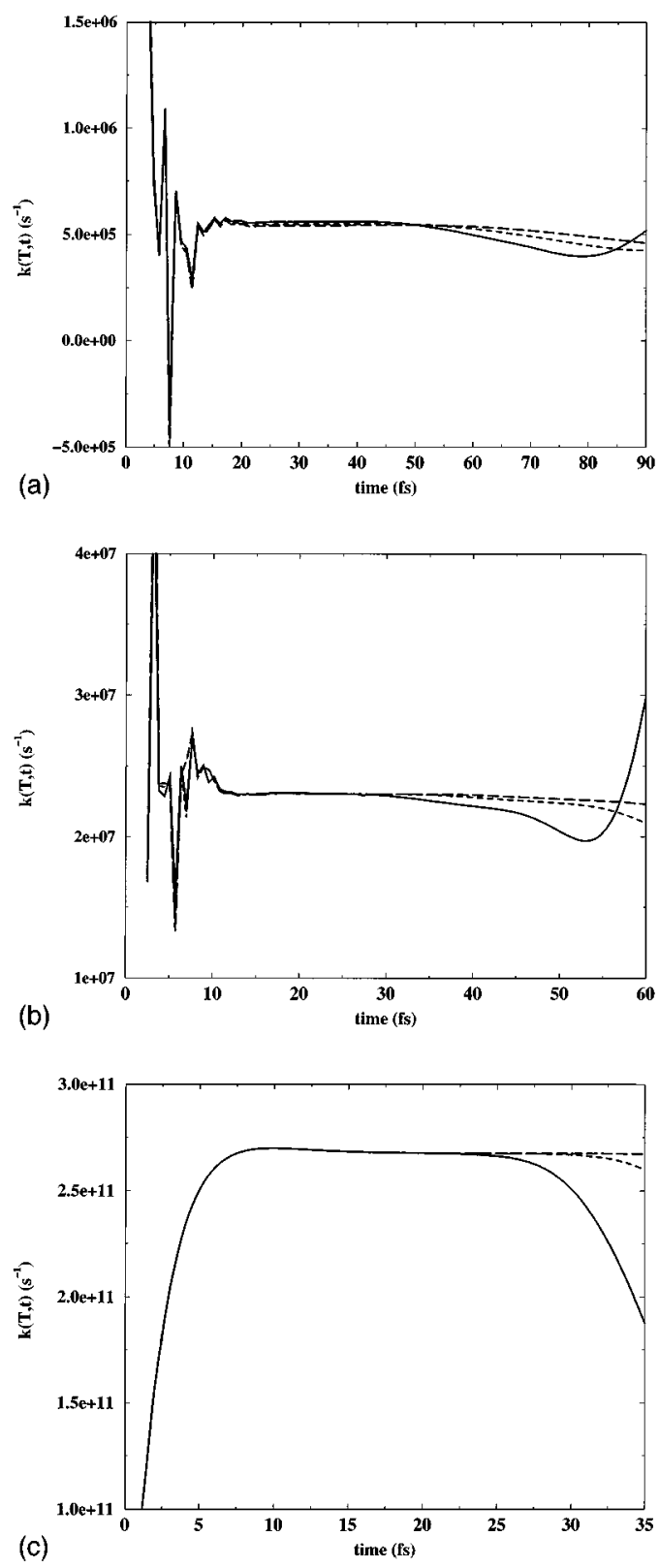

FIG. 1. Thermal rate constants $k(T ; t)$ for the one-dimensional Eckart barrier calculated as a function of time from the flux-position correlation function for (a) $T=200 \mathrm{~K}$, (b) $T=300 \mathrm{~K}$, and (c) $T=1000 \mathrm{~K}$. In (a) results are shown for grid sizes of $Q_{\max }=7.0$ a.u. (solid line), 8.0 a.u. (dashed line), and 9.0 a.u. (long dashed line). In (b) grid sizes of $Q_{\max }=5.0$ a.u. (solid line), 6.0 a.u. (dashed line), and 7.0 a.u. (long dashed line) are shown. And (c) shows results for grid sizes of $Q_{\max }=4.0$ a.u. (solid line), 5.0 a.u. (dashed line), and 6.0 a.u. (long dashed line).

havior has previously been observed by Tromp and Miller, in the flux-flux autocorrelation function. At higher temperatures, the plateau time depends on the temperature-dependent dynamics of crossing the barrier, while at lower temperatures, the rate is dominated by tunneling. However, the tunneling time depends strongly on the barrier frequency but only weakly on the temperature (as shown for the harmonic barrier by Miller, Schwartz, and Tromp ${ }^{3}$ ).

For all temperatures we see that the plateau region may be extended by making the grid larger. This is particularly an issue at lower temperatures. Because we have a Boltzmann distribution of translational energies, at low temperatures there is a contribution from energies above the barrier even though the rate is dominated by tunneling. Reflection from the edges of the grid occurs at these higher energies which cross the barrier at times less than the tunneling time. Thus, lower temperatures require grids which extend farther away from the barrier. In Fig. 1(a), for $T=200 \mathrm{~K}$ with $Q_{\max }=7.0$ a.u., a grid of 31 DVR points is necessary. For $Q_{\max }=8.0$ and 9.0 a.u. at the same temperature, 35 and $39 \mathrm{DVR}$ points are required, respectively. At $T=1000 \mathrm{~K}, 39 \mathrm{DVR}$ points are required for $Q_{\max }=4.0$ a.u., 49 points for $Q_{\max }=5.0$ a.u., and 59 points for $Q_{\max }=6.0$ a.u. We note that the grid sizes necessary for these calculations compare favorably with those used by Seideman and Miller ${ }^{20}$ for direct calculations of the cumulative reaction probability.

For all the results shown, we have used $\eta=1.3$ to determine the number of Chebychev terms. We have found this to give accurate results while minimizing the computational effort. For the results shown for the Eckart barrier in Fig. 1 we have used a maximum of about 230 Chebychev terms (for $1000 \mathrm{~K}$ at the longest times). The number of Lanczos iterations needed is 4 for all but the lowest temperatures. This implies a rank of 2 , as an additional 2 iterations are needed to insure the trace is converged. At lower temperatures, approximately 10 eigenvalues are needed. This is due to our choice of the form of the flux operator. As discussed above, it is possible to express the flux operator as a dyadic, ${ }^{31}$ so $\hat{C}_{f s}(t)$ will be of rank 2 at all temperatures. Equation (3.8) does not guarantee this low rank, but we have chosen to use it because it is more easily applied to higher dimensions.

\section{B. Collinear $\mathbf{H}+\mathrm{H}_{2}$}

The collinear $\mathrm{H}+\mathrm{H}_{2}$ reaction serves as a standard test problem for reactive scattering methods and presents us with the first step to treating multidimensional systems. An accurate potential energy surface exists ${ }^{36}$ and many exact calculations are available for comparison. . $^{11,14,15}$

We have used a DVR grid in the normal mode coordinates $\left(q_{1}, q_{2}\right)$ of the transition state. In these coordinates, the optimum dividing surface is defined by $q_{2}=0$, where $q_{2}$ is the asymmetric stretch and $q_{1}$ is the symmetric stretch normal mode. The raw grid is truncated according to an energy cutoff; if the potential energy at a given DVR point is greater than a specified cutoff energy, $V_{\text {cut }}$, then that DVR point is discarded. The grid is also truncated in the asymptotic reactant and product valleys in the following manner: points are omitted if the translational Jacobi coordinate, $R\left(q_{1}, q_{2}\right)$ is larger than a specified value, $R_{\max }$. The reactant partition function is given by

$$
Q_{r}(T)=\left(\frac{\mu}{2 \pi \hbar^{2} \beta}\right)^{1 / 2} \sum_{v} e^{-\beta \epsilon_{v}},
$$

where $\mu$ is the reduced mass associated with the relative translation of $\mathrm{H}$ and $\mathrm{H}_{2}$. The $\epsilon_{v}$ are the vibrational energy levels of $\mathrm{H}_{2}$ calculated numerically.

Figure 2 shows the time dependence of $C_{f s}(t)$ for different grid sizes for (a) $T=300 \mathrm{~K}$, (b) $T=500 \mathrm{~K}$, and (c) $T=1000 \mathrm{~K}$. At $300 \mathrm{~K}$, the convergence is virtually the same 

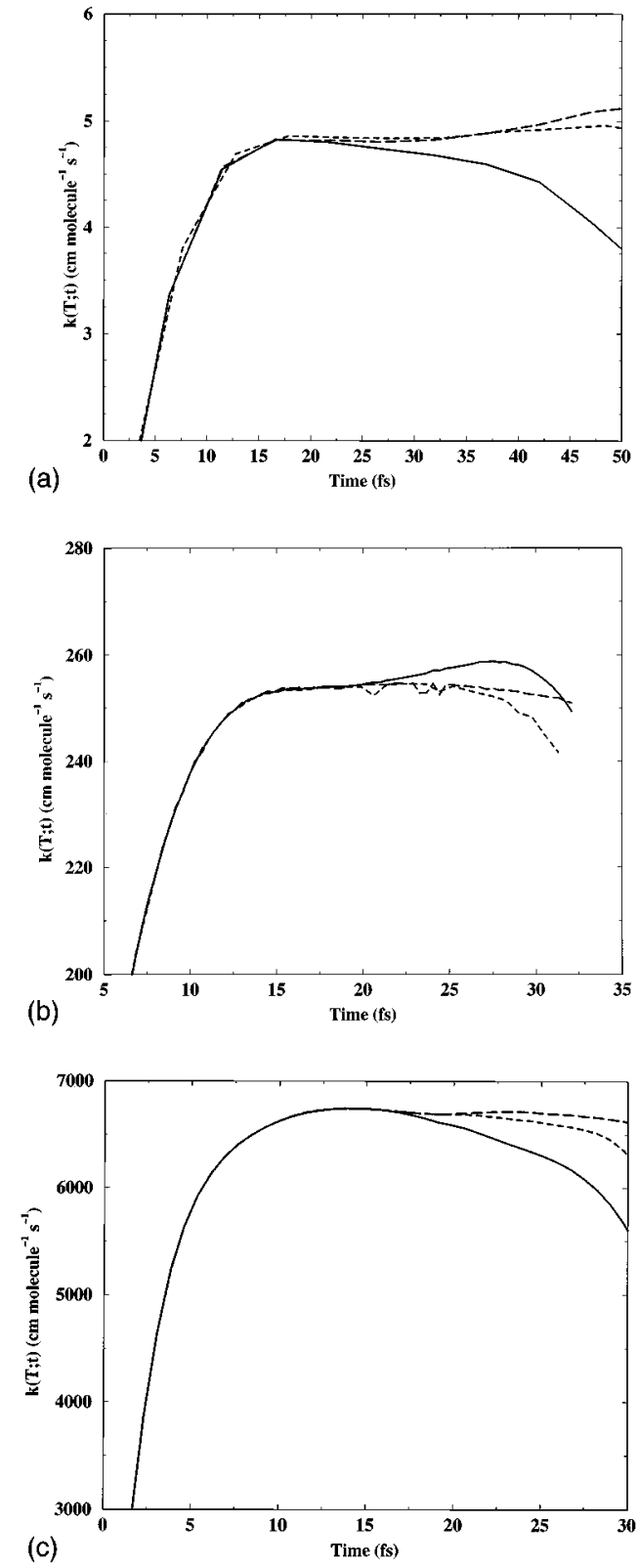

FIG. 2. Thermal rate constants $k(T ; t)$ for the collinear $\mathrm{H}+\mathrm{H}_{2}$ reaction calculated as a function of time from the flux-position correlation function for (a) $T=300 \mathrm{~K}$, (b) $T=500 \mathrm{~K}$, and (c) $T=1000 \mathrm{~K}$. In (a) for $T=300 \mathrm{~K}$ results are shown for grid sizes of $R_{\max }=6.0$ a.u. (solid line), 6.5 a.u. (dashed line), and 7.0 a.u. (long dashed line). In (b) and (c) grid sizes of $R_{\max }=5.0$ a.u. (solid line), 5.5 a.u. (dashed line), and 6.0 a.u. (long dashed line) are shown.

as for the Eckart barrier; the plateau begins around $18 \mathrm{fs}$ and is lengthened by increasing the extent of the grid. For $T=500$ $\mathrm{K}$ and $T=1000 \mathrm{~K}$ the plateau begins around $15 \mathrm{fs}(\hbar \beta \sim 15$ fs) and $13 \mathrm{fs}$, respectively. This is a slightly longer time at $1000 \mathrm{~K}$ than for the Eckart barrier. Again, for the lower temperatures, the time is determined by the tunneling time.

The size of the DVR grid for the results shown varies from 82 points for $R_{\max }=6.0$ at $300 \mathrm{~K}$ to 364 for $R_{\max }=6.0$ and $T=1000 \mathrm{~K}$. Realistically, one needs a grid of around 100 points at $300 \mathrm{~K}, 150$ points for $500 \mathrm{~K}$, and 300 points for $1000 \mathrm{~K}$ to obtain converged results. This is on the order of the size of the basis used by Seideman and Miller ${ }^{20}$ for cal-
TABLE I. Thermal rate constants for the collinear $\mathrm{H}+\mathrm{H}_{2}$ reaction in units of $\mathrm{cm}$ molecule $\mathrm{e}^{-1} \mathrm{~s}^{-1}$.

\begin{tabular}{cccc}
\hline \hline & \multicolumn{3}{c}{$k(T)$} \\
\cline { 2 - 4 } Temp. (K) & Present $^{\mathrm{a}}$ & Ref. 11 & Ref. 15 \\
\hline 300 & 4.82 & 4.821 & 4.82 \\
350 & 18.2 & 18.96 & \\
400 & 54.3 & 54.69 & \\
500 & 254 & 252.9 & 252 \\
600 & 724 & 726.3 & \\
700 & 1576 & 1574 & \\
800 & 2853 & 2848 & \\
900 & 4573 & 4557 & 6680 \\
1000 & 6703 & 6692 & $2.21(4)$ \\
1500 & $2.29(4)^{\mathrm{b}}$ & & $4.20(4)$ \\
2000 & $4.76(4)$ & & \\
\hline \hline
\end{tabular}

${ }^{\mathrm{a}}$ Calculated from Eq. (2.5).

${ }^{\mathrm{b}}$ The number in parentheses is the power of 10 .

culating cumulative reaction probabilities. The number of Chebychev terms needed for the propagation in complex time was less that 700 for all cases. However, around 300 terms are usually sufficient for convergence.

Table I compares the results obtained from the fluxposition correlation function with the results of Rom et al. ${ }^{11}$ and Brown and Light. ${ }^{15}$ The rate constants given are obtained by averaging the results obtained at several times within the plateau period. For all temperatures shown the averaged value is within $1 \%$ of the result for each time within the plateau. We note that at higher temperatures it is necessary to use a larger $V_{\text {cut }}$, as more of the potential energy surface is sampled. As in the case of the Eckart barrier, at lower temperatures we need a larger grid. The agreement between the previous results and our present method is excellent over a wide range of temperatures though our results are higher than those of Brown and Light ${ }^{15}$ above $1000 \mathrm{~K}$.

It is interesting to examine the structure of the eigenvalues of $\hat{C}_{f s}$. Table II shows typical sets of eigenvalues obtained at different temperatures. The pattern is similar to that

TABLE II. Eigenvalues of the $\hat{C}_{f s}$ operator for different temperatures. The eigenvalues have been divided by $Q_{r}(T)$ and are in units of $\mathrm{cm}$ molecule $\mathrm{e}^{-1} \mathrm{~s}^{-1}$. Only eigenvalues with absolute value greater than 0.001 are listed.

\begin{tabular}{rcrrr}
\hline \hline $300 \mathrm{~K}$ & $500 \mathrm{~K}$ & \multicolumn{1}{c}{$1000 \mathrm{~K}$} & \multicolumn{1}{c}{$1500 \mathrm{~K}$} & \multicolumn{1}{c}{$2000 \mathrm{~K}$} \\
\hline 5.9251 & 377.572 & 7759.253 & 23872.925 & 43925.841 \\
0.8528 & 3.508 & 359.960 & 2203.398 & 8866.303 \\
0.4098 & 0.0264 & 16.542 & 51.169 & 1545.173 \\
0.1853 & & 0.990 & 7.421 & 254.994 \\
0.0215 & & 0.0267 & 0.938 & 53.182 \\
0.0035 & & & 0.063 & 19.687 \\
& & & & 0.489 \\
& & & & 0.158 \\
-0.0025 & & & -0.051 & -14.112 \\
-0.0059 & & -0.094 & -3.431 & -41.140 \\
-0.0164 & & -0.462 & -21.274 & -139.343 \\
-0.0876 & -0.0179 & -5.206 & -108.782 & -520.493 \\
-0.1364 & -0.258 & -77.623 & -763.604 & -1233.238 \\
-2.1842 & -122.604 & -1360.776 & -2691.187 & -5244.550 \\
\hline \hline
\end{tabular}


seen by Manthe and Miller ${ }^{28}$ in the eigenreaction probabilities. Namely, as the temperature is raised more eigenvalues contribute to the rate, corresponding to more states of the activated complex which are now energetically (or thermally) accessible. Also, as the temperature increases, the eigenvalues associated with a given state increase, representing an enhancement of the rate through that state. We have a different case than Manthe and Miller ${ }^{28}$ because $\hat{C}_{f s}$ is not a positive definite operator. Therefore we have both positive and negative eigenvalues. The rate is given by the cancellation of the negative eigenvalues by the larger positive ones. This is consistent with the properties of the flux operator discussed above and in more detail by Park and Light. ${ }^{13,14}$

\section{Three dimensional $\mathrm{D}+\mathrm{H}_{2}$ for $J=0$}

Recently there have been several exact ${ }^{16,37}$ and approximate ${ }^{38}$ calculations of the thermal rate constant for the $\mathrm{D}+\mathrm{H}_{2}$ reaction. These provide the opportunity for us to test our method on a full three-dimensional system.

We have calculated the thermal rate constants for the $\mathrm{D}+\mathrm{H}_{2}$ reaction for total angular momentum, $J=0$, on the LSTH (Ref. 36) potential energy surface. We have used a DVR basis in the Jacobi coordinates of the reactant arrangement. We denote the coordinates as $R$, the distance from $D$ to the center-of-mass of $\mathrm{H}_{2}, r$, the $\mathrm{H}_{2}$ bond distance, and $\gamma$, the angle between $\mathbf{R}$ and $\mathbf{r}$. A sinc function DVR is used for $R$ and $r$ while a Gauss-Legendre DVR is used for $\gamma$. We have taken advantage of the inversion symmetry of $\mathrm{H}_{2}$. Thus a separate calculation is done for the even and odd parity blocks of the Hamiltonian; each calculation requires only half of the DVR grid points in the $\gamma$ coordinate. The total rate constant is obtained by adding the even $(p=0)$ and odd parity $(p=1)$ results together with the proper (1:3) nuclear spin weightings,

$$
k(T)=k_{p=0}(T)+3 k_{p=1}(T) .
$$

The same reactant partition function as Mielke et al. ${ }^{37}$ is used,

$$
\begin{aligned}
Q_{r}(T)= & \left(\frac{\mu}{2 \pi \hbar^{2} \beta}\right)^{3 / 2}\left[\sum_{v, j \text { even }}(2 j+1) e^{-\beta \epsilon_{v, j}}\right. \\
& \left.+3 \sum_{v, j \text { odd }}(2 j+1) e^{-\beta \epsilon_{v, j}}\right],
\end{aligned}
$$

where $\mu$ is the reduced mass associated with $R$ and the $\left\{\epsilon_{v, j}\right\}$ are the energy levels of the isolated $\mathrm{H}_{2}$ diatom (calculated numerically).

The basis set is defined by the parameters $N_{B}, N_{\gamma}, p$, $V_{\text {cut }}$, and $R_{\max }$. The grid constant, $N_{B}$, determines the number of points per thermal de Broglie wavelength for the $R$ and $r$ coordinates (See Sec. III D). $N_{\gamma}$ is the number of Gauss-Legendre DVR points used for the $\gamma$ coordinate before symmetrization and $p$ defines the parity of the calculation. If the potential energy at a DVR point is greater than $V_{\text {cut }}$ that point is discarded. The grid is truncated in the asymptotic reactant valley if the translational Jacobi coordinate
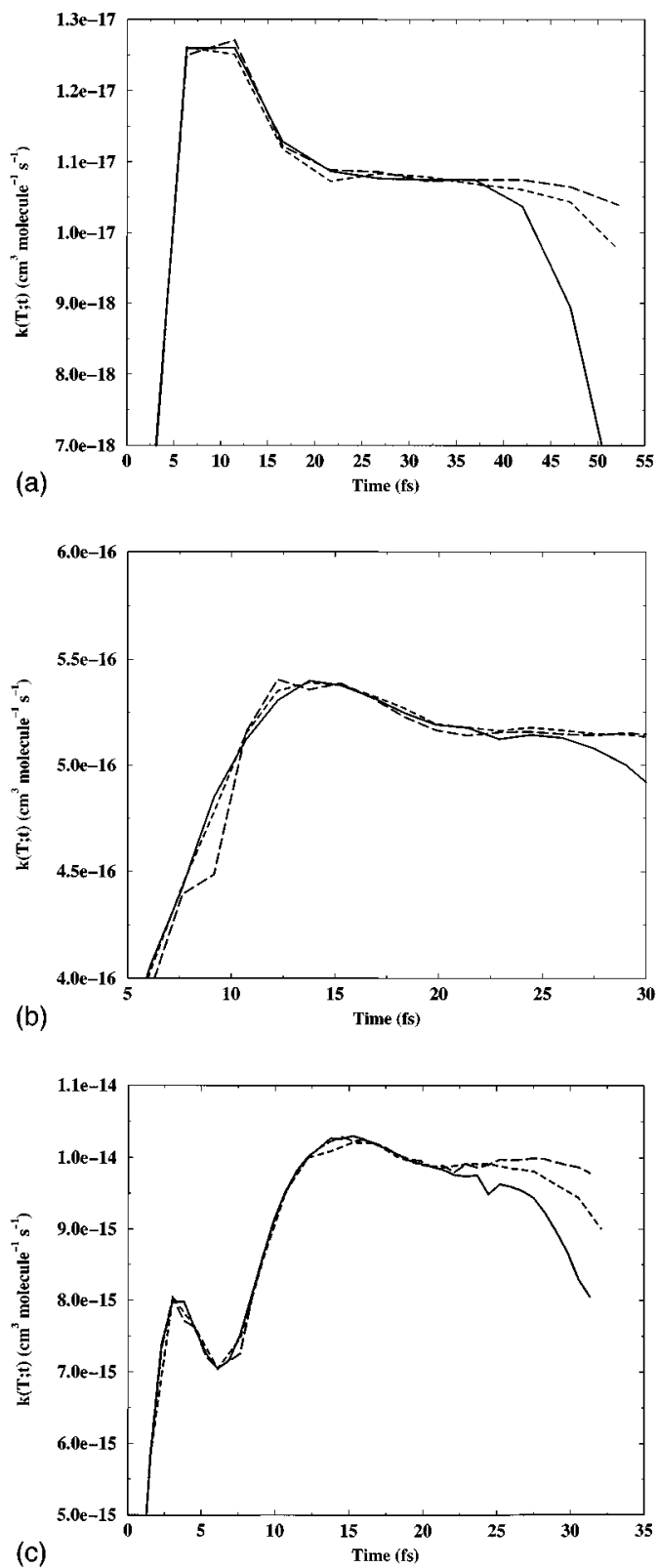

FIG. 3. Thermal rate constants $k(T ; t)$ for the three dimensional $\mathrm{D}+\mathrm{H}_{2}$ reaction calculated as a function of time from the flux-position correlation function for (a) $T=300 \mathrm{~K}$, (b) $T=500 \mathrm{~K}$, and (c) $T=1000 \mathrm{~K}$. In (a) results are shown for grid sizes of $R_{\max }=6.0$ a.u. (solid line), 6.5 a.u. (dashed line), and 7.0 a.u. (long dashed line). In (b) and (c) grid sizes of $R_{\max }=5.0$ a.u. (solid line), 5.5 a.u. (dashed line), and 6.0 a.u. (long dashed line) are shown.

is greater than $R_{\max }$. It is similarly truncated in the product valley by the same criterion, however, the Jacobi coordinates of the product arrangement are used.

Figure 3 illustrates the plateau period for the $\mathrm{D}+\mathrm{H}_{2}$ reaction for (a) $T=300 \mathrm{~K}$, (b) $T=500 \mathrm{~K}$, and (c) $T=1000 \mathrm{~K}$. In this case at $T=300 \mathrm{~K}$ the plateau begins at $22 \mathrm{fs}$ and at $20 \mathrm{fs}$ for $T=500 \mathrm{~K}$, both are slightly longer times than for the collinear $\mathrm{H}+\mathrm{H}_{2}$ case. At $T=1000 \mathrm{~K}$, the plateau begins around $22 \mathrm{fs}$, significantly longer than for collinear $\mathrm{H}+\mathrm{H}_{2}$ or the Eckart barrier. The grid sizes for both temperatures $\left(R_{\max }=6.0\right.$ a.u. for $300 \mathrm{~K}$, and $R_{\max }=5.0$ a.u. for $500 \mathrm{~K}$ and $1000 \mathrm{~K})$ are comparable to those needed for calculating the 
TABLE III. Thermal rate constants for the three-dimensional $\mathrm{D}+\mathrm{H}_{2}(J=0)$ reaction in units of $\mathrm{cm}^{3}$ molecule $\mathrm{s}^{-1} \mathrm{~s}^{-1}$.

\begin{tabular}{cccc}
\hline \hline & \multicolumn{3}{c}{$k(T)$} \\
\cline { 2 - 4 } Temp. (K) & Present $^{\mathrm{a}}$ & Ref. 37 & Ref. 16 \\
\hline 300 & $1.07(-17)$ & $8.17(-18)$ & $9.2(-18)$ \\
500 & $5.15(-16)$ & $5.22(-16)$ & $5.6(-16)$ \\
700 & $2.96(-15)$ & $3.00(-15)$ & $3.2(-15)$ \\
900 & $7.66(-15)$ & $7.59(-15)$ & $8.1(-15)$ \\
1100 & $1.36(-14)$ & $1.33(-14)$ & $1.4(-14)$ \\
1300 & $1.96(-14)$ & $1.94(-14)$ & $2.1(-14)$ \\
1500 & $2.50(-14)$ & $2.53(-14)$ & $2.7(-14)$ \\
\hline \hline
\end{tabular}

${ }^{\mathrm{a} C a l c u l a t e d}$ from Eq. (2.5).

cumulative reaction probability. At the highest temperatures, about 20 Lanczos iterations are needed to converge the rate constant. For all temperatures the number of Chebychev terms necessary is less than 1000 .

Table III compares the results from the flux-position correlation function to the results of Mielke et al. ${ }^{37}$ and Park and Light. ${ }^{16}$ As for the collinear $\mathrm{H}+\mathrm{H}_{2}$ results, the rate constants given in the table are obtained by averaging the results obtained at several times within the plateau period. For all temperatures shown the averaged value is within $2 \%$ of the result for each time within the plateau. The present method gives the rate constant in excellent agreement with the results of Mielke et al. ${ }^{37}$ for temperatures above $300 \mathrm{~K}$. At $300 \mathrm{~K}$ the rate is overestimated by the present method by about $30 \%$.

\section{CONCLUDING REMARKS}

We have introduced a new method for calculating thermal rate constants efficiently and directly via the fluxposition correlation function. This method takes advantage of the low rank of the flux operator to express the thermal rate constant as a sum of the eigenvalues of the thermal reactive flux operator. The eigenvalues are evaluated using a Lanczos scheme which allows the calculation of only those eigenvalues which are nonzero and contribute to the rate. The necessary propagation in complex time, $t_{c}=t-i \hbar \beta / 2$, is accomplished in one step by an efficient Chebychev polynomial expansion. Solving the problem in the time domain avoids the necessity of empirical absorbing potentials. We have tested this new method on three realistic benchmark problems, the one-dimensional Eckart barrier, the twodimensional collinear $\mathrm{H}+\mathrm{H}_{2}$ reaction, and the threedimensional $\mathrm{D}+\mathrm{H}_{2}$ reaction for total angular momentum $J=0$. These applications have demonstrated that a small number of eigenvalues do contribute to the thermal rate constant, and that the present method is indeed efficient and accurate.

Finally, we note very interesting recent work by Manthe $^{39}$ that uses a similar approach to that described here, i.e., a Lanczos procedure to evaluate the trace of the fluxposition correlation function to obtain the thermal rate "directly." There are, though, significant and interesting differences in the specifics of how this is carried out.

\section{ACKNOWLEDGMENTS}

We wish to thank J. Daniel Gezelter for providing the Lanczos code. This work was supported by the Director, Office of Energy Research, Office of Basic Energy Sciences, Chemical Sciences Division of the U.S. Department of Energy under Contract No. DE-AC03-76SF00098. W.H.T. acknowledges support from the National Science Foundation.

${ }^{1}$ W. H. Miller, J. Chem. Phys. 61, 1823 (1974).

${ }^{2}$ S. Chapman, B. C. Garrett, and W. H. Miller, J. Chem. Phys. 63, 2710 (1975)

${ }^{3}$ W. H. Miller, S. D. Schwartz, and J. W. Tromp, J. Chem. Phys. 79, 4889 (1983)

${ }^{4}$ T. Yamamoto, J. Chem. Phys. 33, 281 (1960).

${ }^{5}$ J. W. Tromp and W. H. Miller, J. Phys. Chem. 90, 3482 (1986).

${ }^{6}$ J. W. Tromp and W. H. Miller, Faraday Discuss. Chem. Soc. 84, 441 (1987)

${ }^{7}$ J. W. Tromp, Ph.D. thesis, Lawrence Berkeley Laboratory, University of California, 1988

${ }^{8}$ (a) G. Wahnström and H. Metiu, J. Phys. Chem. 92, 3240 (1988); (b) G. Wahnström, B. Carmeli, and H. Metiu, J. Chem. Phys. 88, 2478 (1988).

${ }^{9}$ (a) M. Thachuk and G. C. Schatz, J. Chem. Phys. 97, 7297 (1992); (b) M. Thachuk, H. R. Mayne, and G. C. Schatz, ibid. 99, 3516 (1993).

${ }^{10}$ P. N. Day and D. G. Truhlar, J. Chem. Phys. 94, 2045 (1991).

${ }^{11}$ N. Rom, N. Moiseyev, and R. Lefebvre, J. Chem. Phys. 96, 8307 (1992).

${ }^{12}$ R. Lefebvre, V. Ryaboy, and N. Moiseyev, J. Chem. Phys. 98, 8601 (1993)

${ }^{13}$ T. J. Park and J. C. Light, J. Chem. Phys. 85, 5870 (1986).

${ }^{14}$ T. J. Park and J. C. Light, J. Chem. Phys. 88, 4897 (1988).

${ }^{15}$ D. Brown and J. C. Light, J. Chem. Phys. 97, 5465 (1992).

${ }^{16}$ T. J. Park and J. C. Light, J. Chem. Phys. 94, 2946 (1991).

${ }^{17}$ (a) T. J. Park and J. C. Light, J. Chem. Phys. 91, 974 (1989); (b) 96, 8853 (1992).

${ }^{18}$ (a) N. Makri and W. H. Miller, J. Chem. Phys. 90, 904 (1989); (b) N. Makri, ibid. 94, 4949 (1991); (c) M. Topaler and N. Makri, ibid. 101, 7500 (1994).

${ }^{19}$ T. N. Truong, J. A. McCammon, D. J. Kouri, and D. K. Hoffman, J. Chem. Phys. 96, 8136 (1992).

${ }^{20}$ T. Seideman and W. H. Miller, J. Chem. Phys. 96, 4412 (1992).

${ }^{21}$ T. Seideman and W. H. Miller, J. Chem. Phys. 97, 2499 (1992); W. H. Miller and T. Seideman, in Time Dependent Quantum Molecular Dynamics: Experiment and Theory, edited by J. Broeckhove (NATO, ARW, 1992).

${ }^{22}$ A. Goldberg and B. W. Shore, J. Phys. B 11, 3339 (1978).

${ }^{23}$ C. Leforestier and R. E. Wyatt, J. Chem. Phys. 78, 2334 (1983).

${ }^{24}$ R. Kosloff and D. Kosloff, J. Comput. Phys. 63, 363 (1986).

${ }^{25}$ D. Neuhauser and M. Baer, J. Chem. Phys. 90, 4351 (1989); 91, 4651 (1989); D. Neuhauser, M. Baer, and D. J. Kouri, ibid. 93, 2499 (1990).

${ }^{26}$ G. Jolicard and E. J. Austin, Chem. Phys. Lett. 121, 106 (1985); G. Jolicard and E. J. Austin, Chem. Phys. 103, 295 (1986); G. Jolicard and M. Y. Perrin, ibid. 116, 1 (1987); G. Jolicard, C. Leforestier, and E. J. Austin, J. Chem. Phys. 88, 1026 (1988)

${ }^{27}$ I. Last, D. Neuhauser, and M. Baer, J. Chem. Phys. 96, 2017 (1992); I. Last and M. Baer, Chem. Phys. Lett. 189, 84 (1992); I. Last, A. Baram, and M. Baer, ibid. 195, 435 (1992); I. Last, A. Baram, H. Szichman, and M. Baer, J. Phys. Chem. 97, 7040 (1993).

${ }^{28}$ U. Manthe and W. H. Miller, J. Chem. Phys. 99, 3411 (1993).

${ }^{29}$ U. Manthe, T. Seideman, and W. H. Miller, J. Chem. Phys. 99, 10078 (1993); 101, 4759 (1994).

${ }^{30}$ D. Thirumalai, B. C. Garrett, and B. J. Berne, J. Chem. Phys. 83, 2972 (1985)

${ }^{31}$ T. Seideman and W. H. Miller, J. Chem. Phys. 95, 1768 (1991).

${ }^{32}$ H. Tal-Ezer and R. Kosloff, J. Chem. Phys. 81, 3967 (1984).

${ }^{33}$ D. O. Harris, G. G. Engerholm, and W. D. Gwinn, J. Chem. Phys. 43, 1515 (1965) 
${ }^{34}$ (a) J. V. Lill, G. A. Parker, and J. C. Light, Chem. Phys. Lett. 89, 483 (1982); (b) J. C. Light, I. P. Hamilton, and J. V. Lill, J. Chem. Phys. 82, 1400 (1985); (c) Z. Bačić and J. C. Light, ibid. 85, 4594 (1986); (d) R. M. Whitnell and J. C. Light, ibid. 89, 3674 (1988); (e) S. E. Choi and J. C. Light, ibid. 92, 2129 (1990).

${ }^{35}$ D. T. Colbert and W. H. Miller, J. Chem. Phys. 96, 1982 (1992).
${ }^{36}$ P. Siegbahn and B. Liu, J. Chem. Phys. 68, 2457 (1978); D. G. Truhlar and C. J. Horowitz, ibid. 68, 2466 (1978).

${ }^{37}$ S. L. Mielke, G. C. Lynch, D. G. Truhlar, and D. W. Schwenke, J. Phys. Chem. 98, 8000 (1994).

${ }^{38}$ D. Wang and J. M. Bowman, J. Phys. Chem. 98, 7994 (1994).

${ }^{39} \mathrm{U}$. Manthe (preprint). 